Supporting information for:

\title{
Synthesis and Application of Chiral Spiro Phospholane Ligand in Pd-Catalyzed Asymmetric Allylation of Aldehydes with Allylic Alcohols
}

\author{
Shou-Fei Zhu, Yun Yang, Li-Xin Wang, Bin Liu and Qi-Lin Zhou * \\ State Key Laboratory and Institute of Elemento-organic Chemistry, Nankai University \\ Tianjin 300071, China
}

\section{CONTENTS}
(A) Preparation and Analytic Data of Ligand $(R)-4$
(B) General Procedure for Asymmetric Allylation of Aldehydes
(C) Analytical Data of Allylation Products
(D) Copies of ${ }^{1} \mathrm{H}$ and ${ }^{13} \mathrm{C}$ NMR Spectra

\section{General:}

All reactions and manipulations which are sensitive to moisture or air were performed in an argon-filled glove box (VAC DRI-LAB HE 493) or using standard Schlenk techniques. Commercial reagents were used as received without further purification unless otherwise noticed. Anhydrous diethyl ether, tetrahydrofuran and toluene were distilled from sodium benzophenone ketyl under nitrogen. Anhydrous DMF were freshly distilled from calcium hydride. $\quad(R)-1,1^{\prime}$-Spirobiindane-7,7'-diol $\quad((R)-5),{ }^{1} \quad(R)-7,7^{\prime}$-bistriflate-1,1'-spirobiindane $((R)-6),{ }^{2}$ were prepared according to the previous papers. Melting points were measured on a RY-I apparatus and uncorrected. NMR spectra were recorded with a Bruker or Varian spectrometer at $300 \mathrm{MHz}\left({ }^{1} \mathrm{H}\right.$ NMR), $75 \mathrm{MHz}\left({ }^{13} \mathrm{C} \mathrm{NMR}\right)$ and $121.5 \mathrm{MHz}\left({ }^{31} \mathrm{P} \mathrm{NMR}\right)$. Chemical shifts were reported in ppm down field from internal $\mathrm{Me}_{4} \mathrm{Si}$ and external $85 \%$ $\mathrm{H}_{3} \mathrm{PO}_{4}$, respectively. Optical rotations were determined using a Perkin Elmer Model 341 
polarimeter. Elemental analyses were performed on Yanaca CDRDER MT-3 instrument. Mass spectra were recorded on a VG-7070E spectrometer. GC analyses were performed using a Hewlett Packard Model HP 6890 Series. HPLC analyses were performed using a Hewlett Packard Model HP 1100 Series or Waters 2996 chromatography.

\section{(A) Preparation and Analytical Data of Ligand (R)-4}

Synthesis of $(R)-7,7^{\prime}$-dicarbonitril-1, $1^{\prime}$-spirobiindane $((R)-7)^{3}$

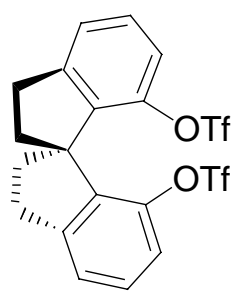

$(R)-6$

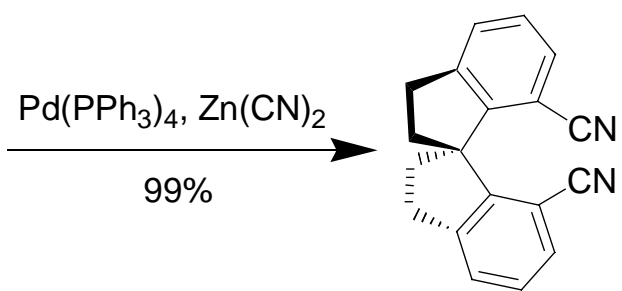

$(R)-7$

A mixture of bistriflate $(R)-6(6.7 \mathrm{~g}, 13 \mathrm{mmol}), \mathrm{Zn}(\mathrm{CN})_{2}(3.6 \mathrm{~g}, 30.1 \mathrm{mmol})$, and $\mathrm{Pd}\left(\mathrm{PPh}_{3}\right)_{4}$ $(1.6 \mathrm{~g}, 1.38 \mathrm{mmol})$ in DMF $(13 \mathrm{~mL})$ was stirred at $140{ }^{\circ} \mathrm{C}$ for 24 hours. The resulting mixture was diluted with ethyl acetate, washed sequentially with aqueous $\mathrm{Na}_{2} \mathrm{CO}_{3}$, saturated brine and dried over anhydrous $\mathrm{MgSO}_{4}$. The dried organic layer was filtrated and concentrated under reduced pressure. The residue was purified by flash chromatography (ethyl acetate/petroleum ether 1:7) to afford $(R)-7(3.5 \mathrm{~g}, 99 \%)$ as a white crystal, mp 194-195 ${ }^{\circ} \mathrm{C} .[\alpha]^{21} \mathrm{D}+144(c 0.5$, $\left.\mathrm{CH}_{2} \mathrm{Cl}_{2}\right) ;{ }^{1} \mathrm{H}$ NMR $\left(300 \mathrm{MHz}, \mathrm{CDCl}_{3}\right) \delta$ 7.54-7.47 (m, 4H, Ar-H), $7.34(\mathrm{t}, J=7.8 \mathrm{~Hz}, 2 \mathrm{H}$, Ar-H), 3.22-3.03 (m, 4H, CH 2$), 2.50-2.30\left(\mathrm{~m}, 4 \mathrm{H}, \mathrm{CH}_{2}\right) ;{ }^{13} \mathrm{C} \mathrm{NMR}\left(75 \mathrm{MHz}, \mathrm{CDCl}_{3}\right) \delta 151.1$, 146.0, 132.0, 129.6, 128.5, 116.7, 108.0, 61.8, 39.6, 31.1; MS (EI) m/z 270 (M+ , 100); Anal. calcd for $\mathrm{C}_{19} \mathrm{H}_{14} \mathrm{~N}_{2}$ : C 84.42, H 5.22, N 10.36; Found: C 84.24, H 5.36, N 10.56.

Synthesis of $(R)-1,1^{\prime}$-spirobiindanyl-7,7'-dicarboxylic acid $((R)-8)$ 


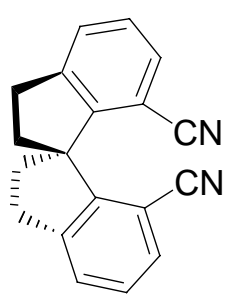

$(R)-7$

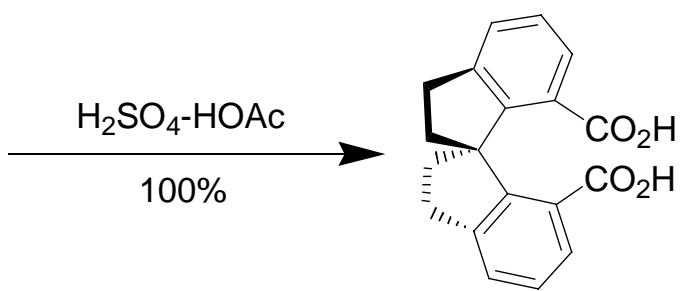

$(R)-8$

The dinitrile $(R)-7(3.5 \mathrm{~g}, 13.0 \mathrm{mmol})$ was added to a mixture of $\mathrm{H}_{2} \mathrm{O}(60 \mathrm{~mL}), \mathrm{HOAc}(30$ $\mathrm{mL})$ and $\mathrm{H}_{2} \mathrm{SO}_{4}(90 \mathrm{~mL})$ and stirred at $145^{\circ} \mathrm{C}$ for 48 hours. The resulting mixture was diluted with water $(600 \mathrm{~mL})$, and extracted with ethyl acetate $(300 \mathrm{~mL})$ for three times. The organic layer was washed with saturated brine, dried over anhydrous $\mathrm{MgSO}_{4}$ and concentrated in reduced pressure. The residue was chromatographied with ethyl acetate/petroleum ether 1:1 to afford $(R)-8(4.0 \mathrm{~g}, 100 \%)$. Further purification by recrystallization with toluene gave a white solid, mp 230-231 ${ }^{\circ} \mathrm{C}$. $[\alpha]^{20}{ }_{\mathrm{D}}+303\left(c\right.$ 0.5, $\left.\mathrm{CH}_{2} \mathrm{Cl}_{2}\right) ;{ }^{1} \mathrm{H}$ NMR $\left(300 \mathrm{MHz}, \mathrm{CDCl}_{3}\right) \delta$ $10.20\left(\mathrm{bs}, 2 \mathrm{H}, \mathrm{CO}_{2} \mathrm{H}\right), 7.63$ (d, J = 7.5 Hz, 2H, Ar-H), 7.39 (d, J= 7.5 Hz, 2H, Ar-H), 7.12 (t, $J=7.5 \mathrm{~Hz}, 2 \mathrm{H}, \mathrm{Ar}-\mathrm{H}), 3.15-2.96\left(\mathrm{~m}, 4 \mathrm{H}, \mathrm{CH}_{2}\right), 2.53-2.21\left(\mathrm{~m}, 4 \mathrm{H}, \mathrm{CH}_{2}\right) ;{ }^{13} \mathrm{C} \mathrm{NMR}(75 \mathrm{MHz}$, $\left.\mathrm{CDCl}_{3}\right) \delta 172.1,151.5,145.5,129.5,128.8,126.5,125.2,63.3,38.2,31.0 ; \mathrm{MS}(\mathrm{EI}) \mathrm{m} / \mathrm{z} 308$ (M+ 6), 290 (100); Anal. calcd for $\mathrm{C}_{19} \mathrm{H}_{16} \mathrm{O}_{4}$ : C 74.01, H 5.23; Found: C 74.07, H 5.40.

\section{Synthesis of $(R)-7,7^{\prime}$-dihydroxymethyl-1,1'-spirobiindane $((R)-9)$}

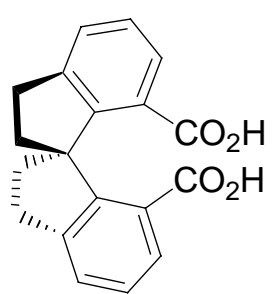

$(R)-8$

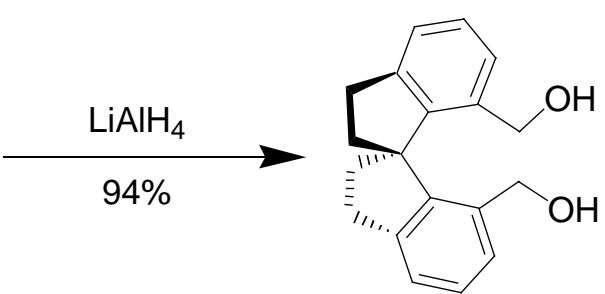

$(R)-9$

To a suspension of $\mathrm{LiAlH}_{4}(2.5 \mathrm{~g}, 64.9 \mathrm{mmol})$ in diethyl ether $(60 \mathrm{~mL})$, a solution of $(R)-8$ $(2.0 \mathrm{~g}, 6.49 \mathrm{mmol})$ in diethyl ether $(120 \mathrm{~mL})$ was added carefully with stirring at $0{ }^{\circ} \mathrm{C}$ within 0.5 hour. After further stirred for 0.5 hour under $0{ }^{\circ} \mathrm{C}$, the system was heated to reflux for 22 hours. After cooling the reaction mixture to ambient temperature, water $(100 \mathrm{~mL})$ and $3 \mathrm{M}$ 
hydrochloric acid $(60 \mathrm{~mL})$ were added slowly. The layers were separated and the aqueous layer was extracted with diethyl ether $(100 \mathrm{~mL})$ for three times. The combined organic layer was washed with water $(100 \mathrm{~mL})$ and brine $(100 \mathrm{~mL})$, dried over anhydrous $\mathrm{Na}_{2} \mathrm{SO}_{4}$, and concentrated in reduced pressure. The residue was chromatographied on silica gel with ethyl acetate/petroleum ether 1:2 to afford $(R)-9(1.7 \mathrm{~g}, 94 \%)$ as a white solid, mp $107-108{ }^{\circ} \mathrm{C}$. $[\alpha]^{21}{ }_{\mathrm{D}}+103\left(c\right.$ 0.5, $\left.\mathrm{CH}_{2} \mathrm{Cl}_{2}\right) ;{ }^{1} \mathrm{H}$ NMR $\left(300 \mathrm{MHz}, \mathrm{CDCl}_{3}\right) \delta$ 7.32-7.20 (m, 6H, Ar-H), 4.22 (dd, $J=22.8$ and $\left.12.3 \mathrm{~Hz}, 4 \mathrm{H}, \mathrm{CH}_{2}\right), 3.05-3.00\left(\mathrm{~m}, 4 \mathrm{H}, \mathrm{CH}_{2}\right), 2.60(\mathrm{~s}, 2 \mathrm{H}, \mathrm{OH}), 2.36-2.29$ (m, $\left.2 \mathrm{H}, \mathrm{CH}_{2}\right), 2.06-1.95\left(\mathrm{~m}, 2 \mathrm{H}, \mathrm{CH}_{2}\right) ;{ }^{13} \mathrm{C} \mathrm{NMR}\left(75 \mathrm{MHz}, \mathrm{CDCl}_{3}\right) \delta$ 147.4, 143.6, 136.7, 128.7, 127.9, 124.4, 61.3, 60.3, 39.7, 31.0; MS (EI) m/z $262\left(\mathrm{M}^{+}-\mathrm{H}_{2} \mathrm{O}, 80\right), 244$ (100); Anal. calcd for $\mathrm{C}_{19} \mathrm{H}_{20} \mathrm{O}_{2}$ : C 81.40, H 7.19; Found: C 81.52, H 7.08.

\section{Synthesis of $(R)-7,7^{\prime}$-dichloromethyl-1,1'-spirobiindane [(R)-10]}

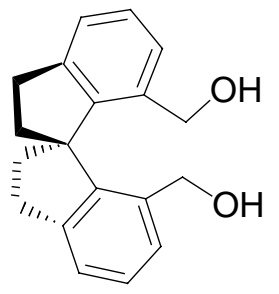

$(R)-9$

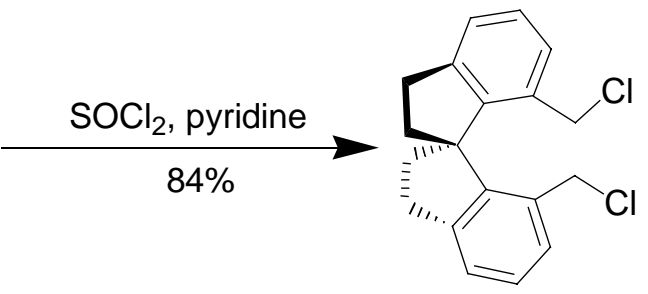

(R)-10

To a solution of $(R)-9(1.45 \mathrm{~g}, 5.17 \mathrm{mmol})$ and pyridine $(0.35 \mathrm{~mL})$ in $\mathrm{CHCl}_{3}(80 \mathrm{~mL})$ was added fresh distilled $\mathrm{SOCl}_{2}(3.8 \mathrm{~mL}, 51.7 \mathrm{mmol})$ in $\mathrm{CHCl}_{3}(40 \mathrm{~mL})$ at $0{ }^{\circ} \mathrm{C}$. After the addition was over, the reaction mixture was stirred at $0^{\circ} \mathrm{C}$ for $30 \mathrm{~min}$ and then heated at reflux for 5 hours. After cooled to ambient temperature, the reaction was then quenched by slow addition of water $(100 \mathrm{~mL})$, and the organic layer was separated. The aqueous layer was extracted with $\mathrm{CHCl}_{3}(100 \mathrm{~mL})$ for three times. The combined organic layer was washed with saturated aqueous $\mathrm{NaHCO}_{3}$, brine, dried over anhydrous $\mathrm{Na}_{2} \mathrm{SO}_{4}$ and concentrated in reduced pressure. The residue was chromatographied on silica gel with ethyl acetate/petroleum ether 1:12 to afford $(R)-\mathbf{1 0}(1.41 \mathrm{~g}, 84 \%)$ as a white solid. Further purification by recrystallization from hexane afforded a colorless crystal, mp 168-169 ${ }^{\circ} \mathrm{C}$. $[\alpha]^{21}{ }_{\mathrm{D}}+230\left(c 0.5, \mathrm{CH}_{2} \mathrm{Cl}_{2}\right) ;{ }^{1} \mathrm{H}$ NMR $\left(300 \mathrm{MHz}, \mathrm{CDCl}_{3}\right) \delta$ 7.29-7.24 (m, 6H, Ar-H), $4.14\left(\mathrm{~d}, J=1.2 \mathrm{~Hz}, 4 \mathrm{H}, \mathrm{CH}_{2}\right)$, 
3.07-3.02 (m, 4H, $\left.\mathrm{CH}_{2}\right), 2.38-2.21\left(\mathrm{~m}, 4 \mathrm{H}, \mathrm{CH}_{2}\right) ;{ }^{13} \mathrm{C} \mathrm{NMR}\left(75 \mathrm{MHz}, \mathrm{CDCl}_{3}\right) \delta 147.2,144.2$, 133.8, 129.9, 128.4, 125.4, 61.6, 42.9, 39.0, 31.0; MS (EI) m/z $316\left(\mathrm{M}^{+}, 14\right), 278$ (100); Anal. calcd for $\mathrm{C}_{19} \mathrm{H}_{18} \mathrm{Cl}_{2}$ : C 71.93, H 5.92; Found: C 71.75, H 5.80.

\section{Phospholane ligand $(R)-4^{4}$}

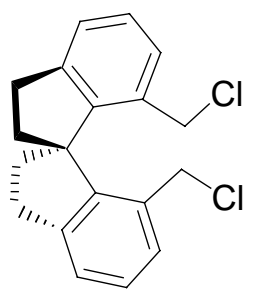

(R)-10

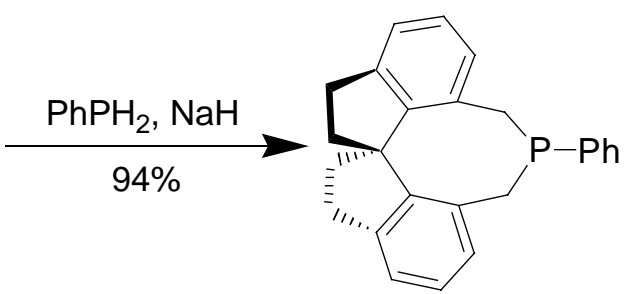

(R)-4

To a suspension of $(R)-\mathbf{1 0}(400 \mathrm{mg}, 1.26 \mathrm{mmol})$ and NaH $(95 \mathrm{mg}, 3.96 \mathrm{mmol})$ in THF (22 ml) was added phenylphosphine $(153 \mathrm{mg}, 1.39 \mathrm{mmol})$ at $-78{ }^{\circ} \mathrm{C}$ under nitrogen. The mixture was stirred at ambient temperature for 24 hours and then heated at refluxing for another 24 hours. After the reaction was completed (monitored by TLC), the solvent was removed in vacuum and the residue was passed through a silica gel plug with ethyl acetate/petroleum ether 1:10 under nitrogen atmosphere to afford the fairly pure product $(\boldsymbol{R})-4(418 \mathrm{mg}, 94 \%)$ as a solid, mp $148-150{ }^{\circ} \mathrm{C} .[\alpha]^{21}{ }_{\mathrm{D}}+6\left(c 0.5, \mathrm{CH}_{2} \mathrm{Cl}_{2}\right) ;{ }^{1} \mathrm{H}$ NMR $\left(300 \mathrm{MHz}, \mathrm{CDCl}_{3}\right) \delta$ 7.31-6.93 (m, 9H, Ar-H), 6.70 (t, $J=7.5 \mathrm{~Hz}, 1 \mathrm{H}$, Ar-H), 5.71 (d, J=7.5 Hz, 1H, Ar-H), 3.12-2.70 (m, 7H, $\left.\mathrm{CH}_{2}\right), 2.58-2.51\left(\mathrm{~m}, 1 \mathrm{H}, \mathrm{CH}_{2}\right), 2.31-2.17\left(\mathrm{~m}, 2 \mathrm{H}, \mathrm{CH}_{2}\right), 1.99-1.73(\mathrm{~m}, 2 \mathrm{H}$, $\left.\mathrm{CH}_{2}\right) ;{ }^{13} \mathrm{C} \mathrm{NMR}\left(75 \mathrm{MHz}, \mathrm{CDCl}_{3}\right) \delta 148.0,146.8,143.4,142.7,132.8,132.6,129.1,128.7$, 128.6, 128.4, 128.2, 128.0, 127.8, 126.4, 123.1, 122.6, 61.5, 38.7, 37.8, 30.9, 30.8, 30.6, 30.0, 29.6, 29.3, 25.7; ${ }^{31} \mathrm{P}$ NMR (121 MHz, $\left.\mathrm{CDCl}_{3}\right) \delta-19.3$ (s); HRMS (FAB) calcd for $\mathrm{C}_{25} \mathrm{H}_{23} \mathrm{P}$ : 355.1599; Found 355.1610.

\section{(B) General Procedure for Asymmetric Allylation of Aldehydes}




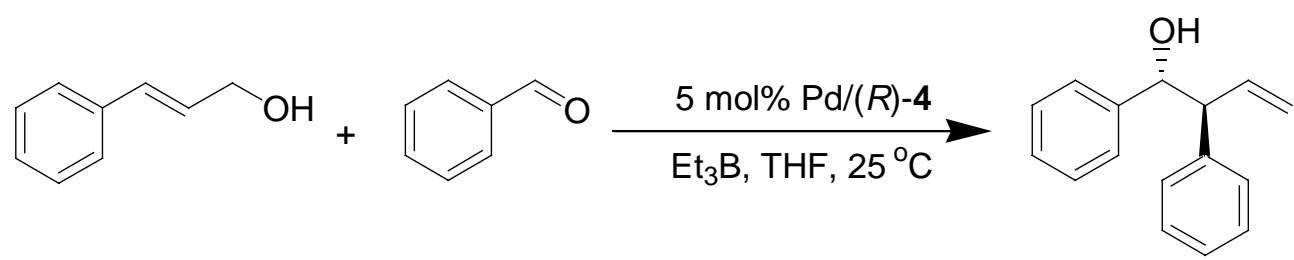

In a Schlenk tube, $\mathrm{Pd}(\mathrm{OAc})_{2}(1.5 \mathrm{mg}, 6.7 \mu \mathrm{mol}),(R)-4(5 \mathrm{mg}, 14.1 \mu \mathrm{mol})$ and THF $(0.8 \mathrm{~mL})$ were added under nitrogen. The reaction mixture was stirred at $25{ }^{\circ} \mathrm{C}$ for $30 \mathrm{~min}$, then the fresh distilled benzaldehyde (18 mg, $1.7 \mathrm{mmol})$, cinnamyl alcohol (19 mg, $1.4 \mathrm{mmol})$ and $\mathrm{Et}_{3} \mathrm{~B}(0.7 \mathrm{~mL}, 1.0 \mathrm{M}$ in hexane, $0.7 \mathrm{mmol})$ were added sequentially. After stirred at $25{ }^{\circ} \mathrm{C}$ for 12 hours, the consuming of cinnamyl alcohol was complete monitored by TLC. The reaction mixture was diluted with ethyl acetate $(40 \mathrm{~mL})$ and hydrolyzed with $3 \mathrm{M}$ hydrochloric acid $(10 \mathrm{~mL})$. After separated with aqueous phase, the organic layer was washed with saturated aqueous $\mathrm{NaHCO}_{3}$, and brine, dried over anhydrous $\mathrm{Na}_{2} \mathrm{SO}_{4}$ and concentrated in reduced pressure. The product was purified by a flash chromatography with ethyl acetate/petroleum ether 1:6 to afford anti-(1R,2S)-1,2-diphenylbut-3-en-1-ol $\mathbf{1 3 a}^{5}$ as a colorless oil. The analytical data of products are listed as follow.

\section{(C) Analytical Data of Allylation Products}

anti-(1R,2S)-1,2-Diphenylbut-3-en-1-ol (13a): ${ }^{5}$ colorless oil, $80 \%$ yield, $82 \%$ ee. $[\alpha]^{18}$ +6.5 (c 0.71, $\left.\mathrm{CHCl}_{3}\right) ;{ }^{1} \mathrm{H} \mathrm{NMR}\left(300 \mathrm{MHz}, \mathrm{CDCl}_{3}\right) \delta$ 7.26-7.04 (m, 10H, Ar-H), 6.26 (ddd, J $=16.8,10.2$ and $8.7 \mathrm{~Hz}, 1 \mathrm{H}, \mathrm{CH}), 5.29-5.21\left(\mathrm{~m}, 2 \mathrm{H}, \mathrm{CH}_{2}\right), 4.85(\mathrm{dd}, J=7.8$ and $2.4 \mathrm{~Hz}, 1 \mathrm{H}$, $\mathrm{CH}), 3.55(\mathrm{t}, J=8.4 \mathrm{~Hz}, 1 \mathrm{H}, \mathrm{CH}), 2.30(\mathrm{~d}, J=2.7 \mathrm{~Hz}, 1 \mathrm{H}, \mathrm{OH}) ;{ }^{13} \mathrm{C} \mathrm{NMR}\left(75 \mathrm{MHz}, \mathrm{CDCl}_{3}\right)$ $\delta 142.1,140.9,138.1,128.6,128.2,127.7,126.9,118.7,77.5,59.5 ; \mathrm{MS}(\mathrm{EI}) \mathrm{m} / \mathrm{z} 224\left(\mathrm{M}^{+}, 2\right)$, 107 (100); HPLC (Chiralpak AD-H column, $n$-Hexane/2-PrOH = 98:2, $0.9 \mathrm{~mL} / \mathrm{min}$ ) $t_{\mathrm{R}}=24.6$ $\min \left(\right.$ minor), $t_{\mathrm{R}}=27.5 \min$ (major).

anti-1-(4-Methoxyphenyl)-2-phenylbut-3-en-1-ol (13b): ${ }^{6}$ colorless oil, $97 \%$ yield, 74\% ee. $[\alpha]^{19}{ }_{\mathrm{D}}+6.8\left(c 0.77, \mathrm{CH}_{2} \mathrm{Cl}_{2}\right) ;{ }^{1} \mathrm{H} \mathrm{NMR}\left(300 \mathrm{MHz}, \mathrm{CDCl}_{3}\right) \delta 7.25-7.03(\mathrm{~m}, 7 \mathrm{H}, \mathrm{Ar}-\mathrm{H})$, 6.74-6.71 (m, 2H, Ar-H), 6.25 (ddd, $J=16.8,10.2$ and $9.0 \mathrm{~Hz}, 1 \mathrm{H}, \mathrm{CH}), 5.28-5.21(\mathrm{~m}, 2 \mathrm{H}$, 
$\left.\mathrm{CH}_{2}\right), 4.80(\mathrm{dd}, J=8.1$ and $2.4 \mathrm{~Hz}, 1 \mathrm{H}, \mathrm{CH}), 3.74\left(\mathrm{~s}, 3 \mathrm{H}, \mathrm{CH}_{3}\right), 3.52(\mathrm{t}, J=8.4 \mathrm{~Hz}, 1 \mathrm{H}, \mathrm{CH})$, $2.29(\mathrm{~s}, 1 \mathrm{H}, \mathrm{OH}) ;{ }^{13} \mathrm{C} \mathrm{NMR}\left(75 \mathrm{MHz}, \mathrm{CDCl}_{3}\right) \delta 159.0,140.9,138.4,134.2,129.0,128.6$, 128.1, 126.8, 118.5, 113.5, 77.0, 59.6, 55.4; MS (EI) m/z $254\left(\mathrm{M}^{+}, 1\right), 137$ (100); HPLC (Chiralpak AD-H column, $n$-Hexane $/ 2-\mathrm{PrOH}=98: 2,0.9 \mathrm{~mL} / \mathrm{min}$ ) $t_{\mathrm{R}}=27.3 \mathrm{~min}$ (major), $t_{\mathrm{R}}=$ $31.4 \mathrm{~min}$ (minor).

anti-1-(2-Methoxyphenyl)-2-phenylbut-3-en-1-ol (13c): colorless oil, 91\% yield, 69\% ee. $[\alpha]^{20}{ }_{\mathrm{D}}+5\left(c\right.$ 0.12, $\left.\mathrm{CH}_{2} \mathrm{Cl}_{2}\right) ;{ }^{1} \mathrm{H}$ NMR $\left(300 \mathrm{MHz}, \mathrm{CDCl}_{3}\right) \delta$ 7.17-7.07 (m, 7H, Ar-H), 6.79-6.68 (m, 2H, Ar-H), 6.22 (ddd, $J=17.1,10.2$ and $8.4 \mathrm{~Hz}, 1 \mathrm{H}, \mathrm{CH}), 5.14-4.98$ (m, 3H, $\mathrm{CH}$ and $\left.\mathrm{CH}_{2}\right), 3.72-3.65\left(\mathrm{~m}, 4 \mathrm{H}, \mathrm{CH}\right.$ and $\left.\mathrm{CH}_{3}\right), 2.59(\mathrm{~d}, J=6.0 \mathrm{~Hz}, 1 \mathrm{H}, \mathrm{OH}) ;{ }^{13} \mathrm{C} \mathrm{NMR}(75$ $\left.\mathrm{MHz}, \mathrm{CDCl}_{3}\right) \delta 156.4,141.7,138.0,130.2,128.3,128.1,128.0,126.3,120.4,117.6,110.3$, 73.9, 56.7, 55.2; MS (EI) m/z $254\left(\mathrm{M}^{+}, 1\right), 137$ (100); Anal. calcd for $\mathrm{C}_{17} \mathrm{H}_{18} \mathrm{O}_{2}: \mathrm{C} 80.28, \mathrm{H}$ 7.13; Found: C 80.35, H 6.97. HPLC (Chiralpak AD-H column, $n$-Hexane/2-PrOH = 95:5, $0.9 \mathrm{~mL} / \mathrm{min}$ ) $t_{\mathrm{R}}=22.0 \mathrm{~min}$ (minor), $t_{\mathrm{R}}=26.5 \mathrm{~min}$ (major).

anti-1-(4-Trifluoromethylphenyl)-2-phenylbut-3-en-1-ol (13d): colorless oil, 71\% yield, $58 \%$ ee. $[\alpha]^{20}{ }_{\mathrm{D}}+2.9\left(c\right.$ 0.99, $\left.\mathrm{CH}_{2} \mathrm{Cl}_{2}\right) ;{ }^{1} \mathrm{H}$ NMR $\left(300 \mathrm{MHz}, \mathrm{CDCl}_{3}\right) \delta$ 7.47-7.03 (m, 9H, Ar-H), 6.23 (ddd, $J=17.1,10.2$ and $9 \mathrm{~Hz}, 1 \mathrm{H}, \mathrm{CH}), 5.32-5.22\left(\mathrm{~m}, 2 \mathrm{H}, \mathrm{CH}_{2}\right), 4.89$ (dd, $J=$ 7.8 and $2.4 \mathrm{~Hz}, 1 \mathrm{H}, \mathrm{CH}), 3.50(\mathrm{t}, J=8.4 \mathrm{~Hz}, 1 \mathrm{H}, \mathrm{CH}), 2.40(\mathrm{~d}, J=2.4 \mathrm{~Hz}, 1 \mathrm{H}, \mathrm{OH}) ;{ }^{13} \mathrm{C}$ $\operatorname{NMR}\left(75 \mathrm{MHz}, \mathrm{CDCl}_{3}\right) \delta 145.7,139.9,137.2,128.6,128.2,127.0,124.9,124.8,119.2,59.4$; MS (EI) m/z $292\left(\mathrm{M}^{+}, 6\right), 118$ (100); Anal. calcd for $\mathrm{C}_{17} \mathrm{H}_{15} \mathrm{~F}_{3} \mathrm{O}$ : C 69.85, H 5.17; Found: C 69.72, H 5.12. HPLC (Chiralpak AD-H column, $n$-Hexane $/ 2-\mathrm{PrOH}=98: 2,0.9 \mathrm{~mL} / \mathrm{min}$ ) $t_{\mathrm{R}}=$ $14.9 \min$ (major), $t_{\mathrm{R}}=16.8 \min$ (minor).

anti-1-(1-Naphtyl)-2-phenylbut-3-en-1-ol (13e): pale yellow oil, 99\% yield, 79\% ee. $[\alpha]^{15}{ }_{\mathrm{D}}+9\left(c\right.$ 0.45, $\left.\mathrm{CH}_{2} \mathrm{Cl}_{2}\right) ;{ }^{1} \mathrm{H}$ NMR $\left(300 \mathrm{MHz}, \mathrm{CDCl}_{3}\right) \delta$ 8.09-8.06 (m, 1H, Ar-H), 7.85-7.74 (m, 2H, Ar-H), 7.53-7.36 (m, 4H, Ar-H), 7.24-7.12 (m, 5H, Ar-H), 6.32 (ddd, $J=$ 16.5, 10.2 and $8.4 \mathrm{~Hz}, 1 \mathrm{H}, \mathrm{CH}), 5.67(\mathrm{dd}, J=5.1$ and $3.3 \mathrm{~Hz}, 1 \mathrm{H}, \mathrm{CH}), 5.21(\mathrm{dd}, J=10.2$ and $\left.1.2 \mathrm{~Hz}, 1 \mathrm{H}, \mathrm{CH}_{2}\right), 4.99\left(\mathrm{dd}, J=17.1\right.$ and $\left.1.2 \mathrm{~Hz}, 1 \mathrm{H}, \mathrm{CH}_{2}\right), 3.91(\mathrm{dd}, J=8.7$ and $5.4 \mathrm{~Hz}, 1 \mathrm{H}$, $\mathrm{CH}), 2.32(\mathrm{~d}, J=3.3 \mathrm{~Hz}, 1 \mathrm{H}, \mathrm{OH}) ;{ }^{13} \mathrm{C} \mathrm{NMR}\left(75 \mathrm{MHz}, \mathrm{CDCl}_{3}\right) \delta 141.7,137.7,136.7,133.7$, 
$130.5,129.0,128.5,128.2,128.1,126.7,125.9,125.3,125.1,124.6,123.2,118.6,74.2,56.6$; MS (EI) m/z $274\left(\mathrm{M}^{+}, 6\right), 157$ (100); Anal. calcd for $\mathrm{C}_{17} \mathrm{H}_{18} \mathrm{O}_{2}$ : C 87.56, H 6.61; Found: C 87.69, H 6.77. HPLC (Chiralpak AD-H column, $n$-Hexane $/ 2-\mathrm{PrOH}=98: 2,0.9 \mathrm{~mL} / \mathrm{min}$ ) $t_{\mathrm{R}}=$ $39.9 \min$ (minor), $t_{\mathrm{R}}=57.2 \min$ (major).

anti-1-(2-Naphtyl)-2-phenylbut-3-en-1-ol (13f): colorless oil, 95\% yield, $73 \%$ ee. $[\alpha]^{15}$ -6 (c 0.54, $\left.\mathrm{CH}_{2} \mathrm{Cl}_{2}\right) ;{ }^{1} \mathrm{H}$ NMR $\left(300 \mathrm{MHz}, \mathrm{CDCl}_{3}\right) \delta$ 7.78-7.07 (m, 12H, Ar-H), 6.29 (ddd, $J=$ 17.1, 10.2 and $9.0 \mathrm{~Hz}, 1 \mathrm{H}, \mathrm{CH}), 5.29-5.21\left(\mathrm{~m}, 2 \mathrm{H}, \mathrm{CH}_{2}\right), 5.02(\mathrm{dd}, J=7.8$ and $2.4 \mathrm{~Hz}, 1 \mathrm{H}$, $\mathrm{CH}), 3.66(\mathrm{t}, J=8.1 \mathrm{~Hz}, 1 \mathrm{H}, \mathrm{CH}), 2.41(\mathrm{~d}, J=2.7 \mathrm{~Hz}, 1 \mathrm{H}, \mathrm{OH}) ;{ }^{13} \mathrm{C} \mathrm{NMR}\left(75 \mathrm{MHz}, \mathrm{CDCl}_{3}\right)$ $\delta 140.6,139.3,137.8,133.0,132.9,128.4,128.3,128.0,127.1,126.7,125.9,124.7,118.6$, 77.3, 59.1; MS (EI) m/z $274\left(\mathrm{M}^{+}, 3\right), 157$ (100); Anal. calcd for $\mathrm{C}_{17} \mathrm{H}_{18} \mathrm{O}_{2}$ : C 87.56, H 6.61; Found: C 87.35, H 6.58. HPLC (Chiralpak AD-H column, $n$-Hexane/2-PrOH = 95:5, 0.9 $\mathrm{mL} / \mathrm{min}$ ) $t_{\mathrm{R}}=28.5 \min \left(\right.$ minor),$t_{\mathrm{R}}=30.0$ min (major).

anti-1-(2-Furyl)-2-phenylbut-3-en-1-ol (13g): colorless oil, 73\% yield, $78 \%$ ee. $[\alpha]^{15}$ +47 (c 0.19, $\left.\mathrm{CH}_{2} \mathrm{Cl}_{2}\right) ;{ }^{1} \mathrm{H}$ NMR $\left(300 \mathrm{MHz}, \mathrm{CDCl}_{3}\right) \delta$ 7.32-7.13 (m, 6H, Ar-H), 6.28-6.16 (m, 2H, Ar-H), 6.01 (d, $J=3.0 \mathrm{~Hz}, 1 \mathrm{H}, \mathrm{CH}), 5.30-5.23\left(\mathrm{~m}, 2 \mathrm{H}, \mathrm{CH}_{2}\right), 4.89$ (dd, $J=8.1$ and 3.9 $\mathrm{Hz}, 1 \mathrm{H}, \mathrm{CH}), 3.84(\mathrm{t}, J=8.1 \mathrm{~Hz}, 1 \mathrm{H}, \mathrm{CH}), 2.27(\mathrm{~d}, J=3.9 \mathrm{~Hz}, 1 \mathrm{H}, \mathrm{OH}) ;{ }^{13} \mathrm{C}$ NMR $(75 \mathrm{MHz}$, $\left.\mathrm{CDCl}_{3}\right) \delta 154.1,141.9,140.3,137.6,128.4,128.1,126.8,118.6,110.1,107.6,70.9,55.9 ; \mathrm{MS}$ (EI) m/z $214\left(\mathrm{M}^{+}, 2\right), 39$ (100); Anal. calcd for $\mathrm{C}_{14} \mathrm{H}_{14} \mathrm{O}_{2}$ : C 78.48, H 6.59; Found: C 78.29, H 6.62. HPLC (Chiralpak AD-H column, $n$-Hexane $/ 2-\mathrm{PrOH}=98: 2,0.9 \mathrm{~mL} / \mathrm{min}$ ) $t_{\mathrm{R}}=20.2$ $\min \left(\right.$ major), $t_{\mathrm{R}}=21.6 \min (\operatorname{minor})$.

anti-1-Cyclohexyl-2-phenylbut-3-en-1-ol (13h): ${ }^{7}$ colorless oil, $50 \%$ yield, $83 \%$ ee. $[\alpha]^{18}$ $+38\left(c\right.$ 0.52, $\left.\mathrm{CH}_{2} \mathrm{Cl}_{2}\right) ;{ }^{1} \mathrm{H}$ NMR $\left(300 \mathrm{MHz}, \mathrm{CDCl}_{3}\right) \delta$ 7.35-7.20 (m, 5H, Ar-H), 6.14 (ddd, J = $17.1,8.1$ and $6.9 \mathrm{~Hz}, 1 \mathrm{H}, \mathrm{CH}), 5.22-5.16\left(\mathrm{~m}, 2 \mathrm{H}, \mathrm{CH}_{2}\right), 3.57-3.43(\mathrm{~m}, 1 \mathrm{H}, \mathrm{CH}), 1.81-1.01(\mathrm{~m}$, $12 \mathrm{H}, \mathrm{OH}$ and $\left.\mathrm{CH}_{2}\right) ;{ }^{13} \mathrm{C} \mathrm{NMR}\left(75 \mathrm{MHz}, \mathrm{CDCl}_{3}\right) \delta 142.1,138.4,128.7,128.0,126.5,117.7$, 78.1, 53.7, 39.6, 30.2, 26.5, 26.4, 26.0; MS (EI) m/z 117 (98), 95 (100);HPLC (Chiralpak AD-H column, $n$-Hexane $/ 2-\mathrm{PrOH}=98: 2,0.9 \mathrm{~mL} / \mathrm{min}$ ) $t_{\mathrm{R}}=11.4 \mathrm{~min}$ (major), $t_{\mathrm{R}}=13.3 \mathrm{~min}$ (minor). 
(R)-1-phenylbut-3-en-1-ol (16): ${ }^{8}$ colorless oil, $80 \%$ yield, $80 \%$ ee. $[\alpha]^{15}{ }^{15}+22(c 0.14$, $\left.\mathrm{CH}_{2} \mathrm{Cl}_{2}\right) ;{ }^{1} \mathrm{H}$ NMR $\left(300 \mathrm{MHz}, \mathrm{CDCl}_{3}\right) \delta$ 7.35-7.24 (m, 5H, Ar-H), 5.89-5.75 (m, 1H, CH), 5.19-5.11 (m, 2H, $\left.\mathrm{CH}_{2}\right), 4.74-4.69(\mathrm{~m}, 1 \mathrm{H}, \mathrm{CH}), 2.53-2.47\left(\mathrm{~m}, 2 \mathrm{H}, \mathrm{CH}_{2}\right), 2.16(\mathrm{~s}, 1 \mathrm{H}, \mathrm{OH})$; ${ }^{13} \mathrm{C}$ NMR $\left(75 \mathrm{MHz}, \mathrm{CDCl}_{3}\right) \delta 144.1,134.7,128.7,127.8,126.1,118.7,73.5,44.1$; MS (EI) m/z $148\left(\mathrm{M}^{+}, 8\right), 107$ (100); HPLC (Chiralpak AD-H column, $n$-Hexane/2-PrOH = 98:2, 1.0 $\mathrm{mL} / \mathrm{min}$ ) $t_{\mathrm{R}}=17.8 \min$ (major), $t_{\mathrm{R}}=18.7 \min$ (minor).

\section{Reference}

${ }^{1}$ Zhang, J.-H.; Liao, J.; Cui, X.; Yu, K.-B.; Deng, J.-G.; Zhu, S.-F.; Wang, L.-X.; Zhou, Q.-L.; Chung, L.-W.; Ye, T. Tetrahedron: Asymmtry 2002, 13, 1363.

${ }^{2}$ Xie, J.-H.; Wang, L.-X.; Fu, Y.; Zhu, S.-F.; Fan, B.-M.; Duan, H.-F.; Zhou, Q.-L. J. Am. Chem. Soc. 2003, 125, 4404.

${ }^{3}$ Tuyet, T. M. T.; Harada, T.; Hashimoto, K.; Hatsuda, M.; Oku, A. J. Org. Chem. 2000, 65, 1335.

${ }^{4}$ Xiao, D.-M.; Zhang, Z.-G.; Zhang, X.-M. Org. Lett. 1999, 1, 1679.

${ }^{5}$ Hafner, A.; Duthaler, R. O.; Marti, R.; Rihs, G.; Rothe-Streit, P.; Schwarzenbach, F. J. Am. Chem. Soc. 1992, 114, 2321.

${ }^{6}$ Coxon, J. M.; Eyk, S. J. V.; Steel, P. J. Tetrahedron 1989, 45, 1029.

${ }^{7}$ Lombardo, M.; Morganti, S.; Tozzi, M.; Trombini, C. Eur. J. Org. Chem. 2002, 16, 2823.

${ }^{8}$ (a) Corey, E. J.; Kim, S. S. Tetrahedron Lett. 1990, 31, 3715. $\quad$ (b) Denmark, S. E.; Coe, D. M.; Pratt, N. E.; Griedel, B. D. J. Org. Chem. 1994, 59, 6161.. 
(D) Copies of ${ }^{1} \mathrm{H}$ and ${ }^{13} \mathrm{C}$ NMR Spectra

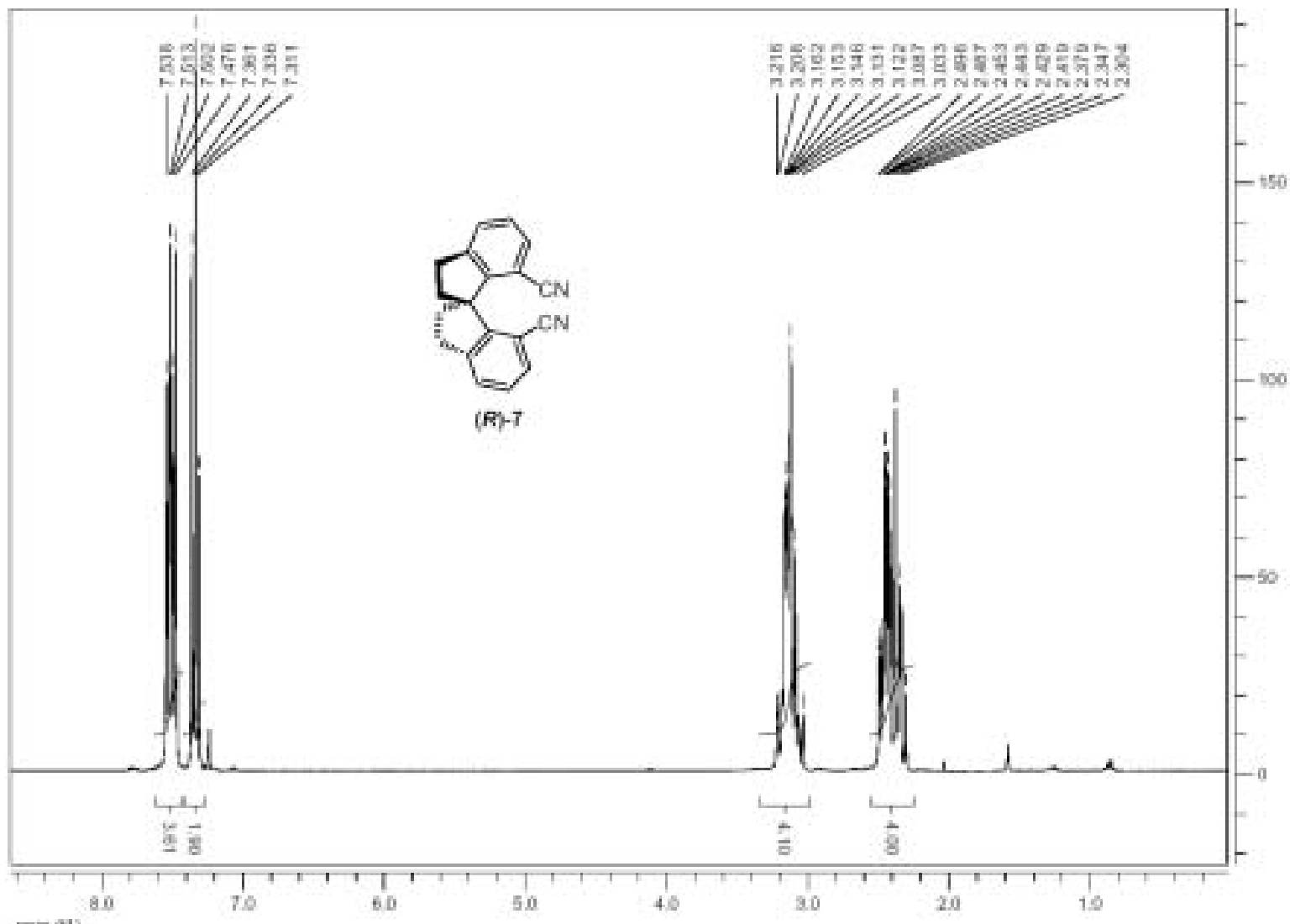

(क्) त)

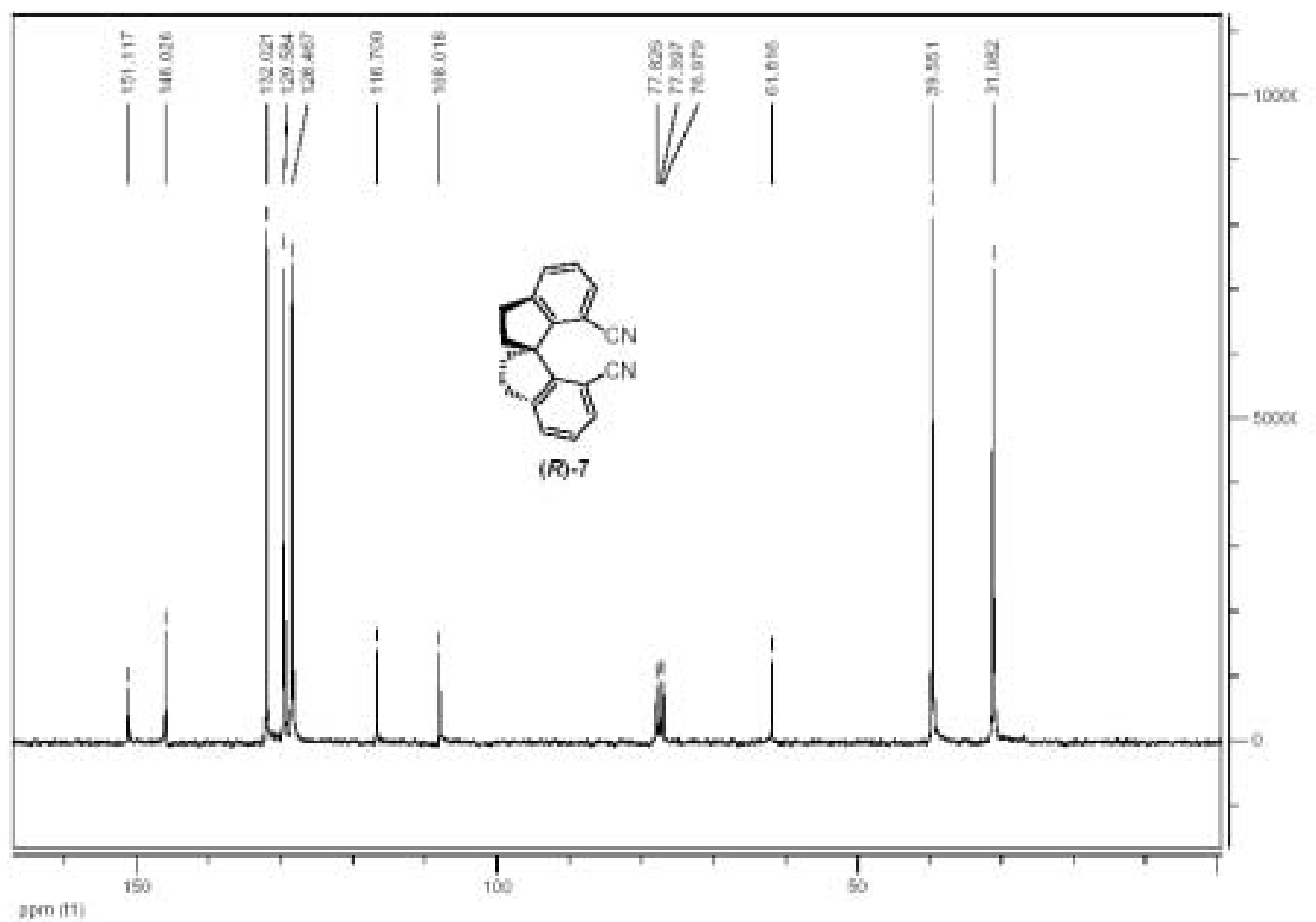




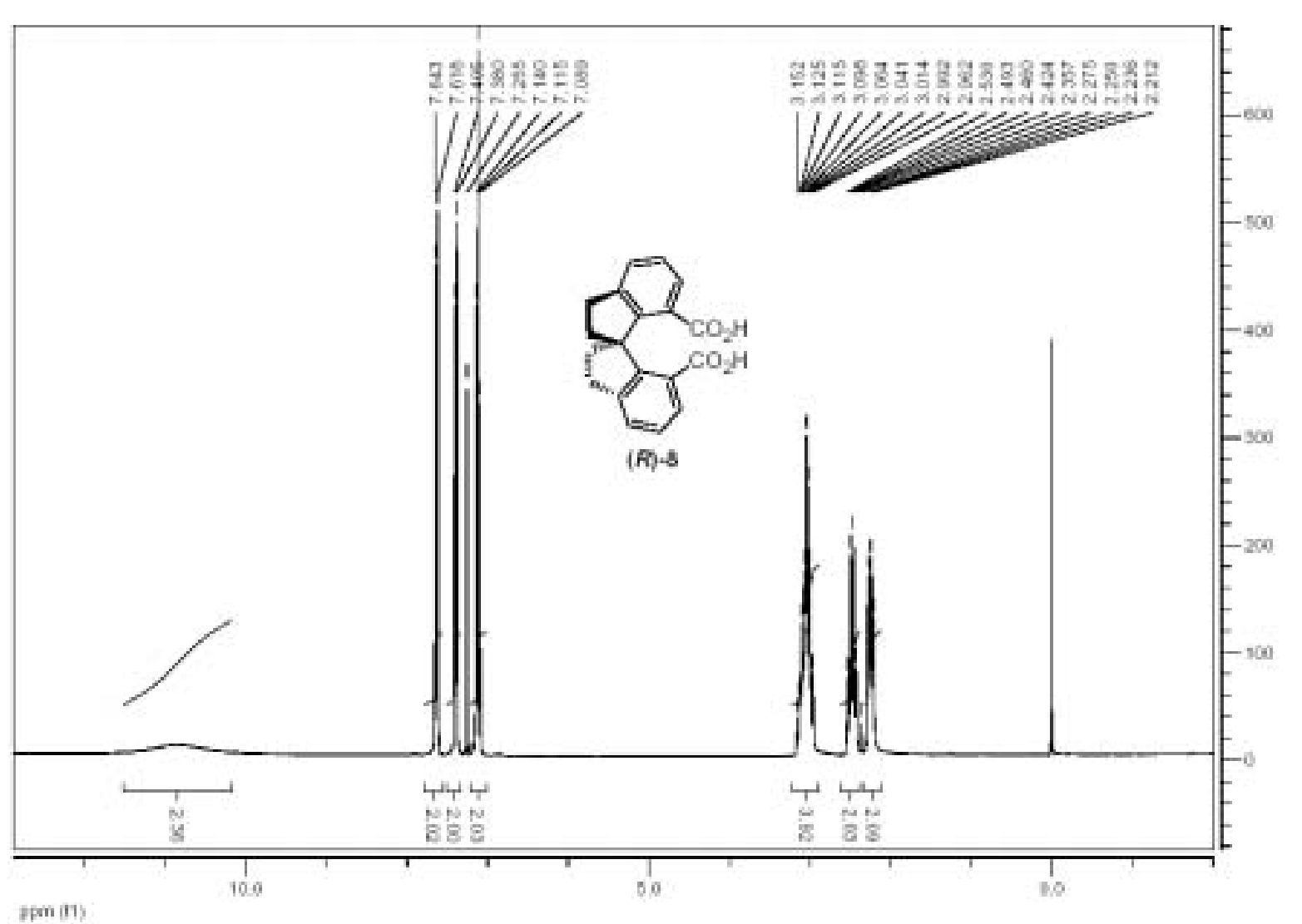

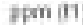

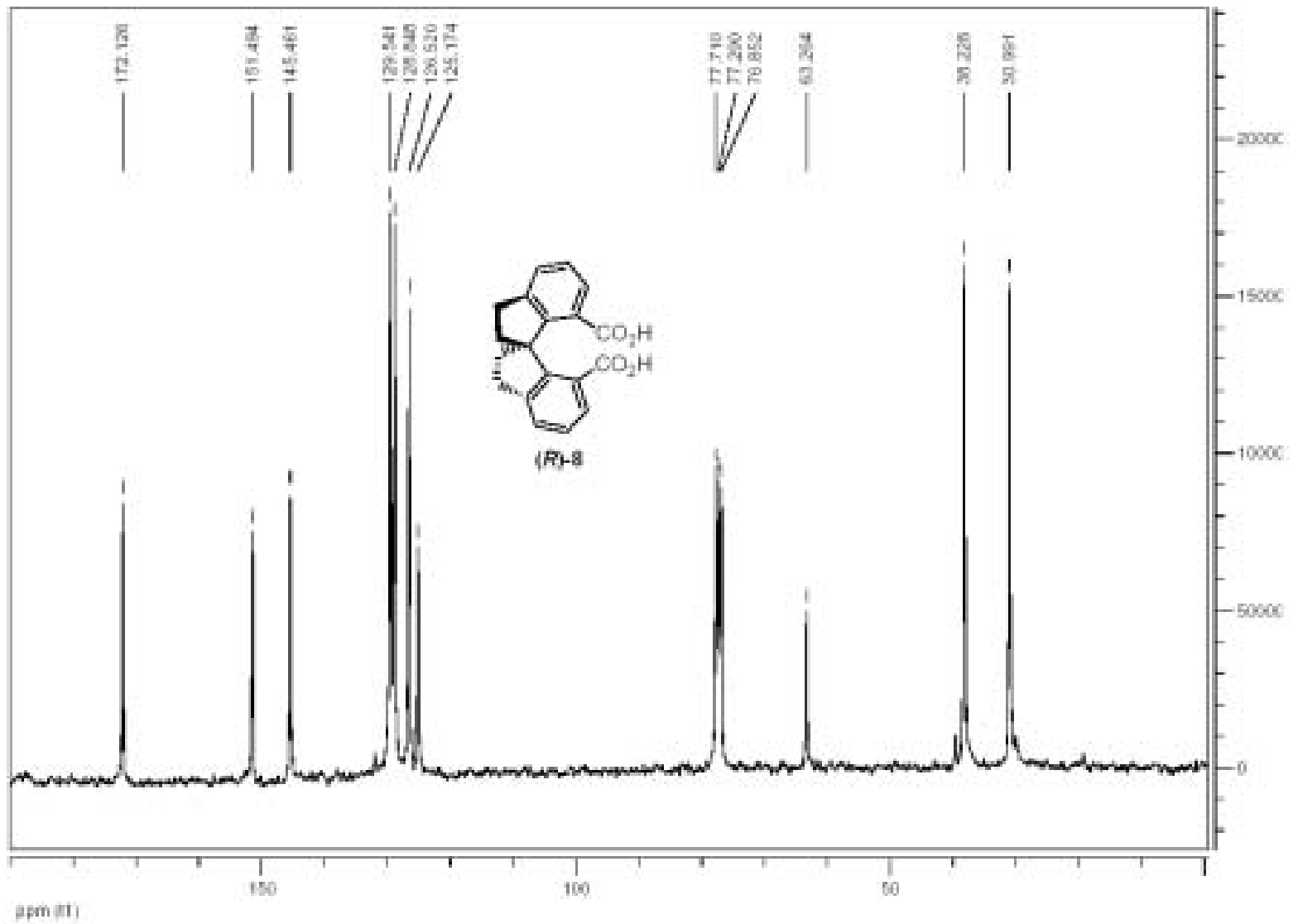

aponti 

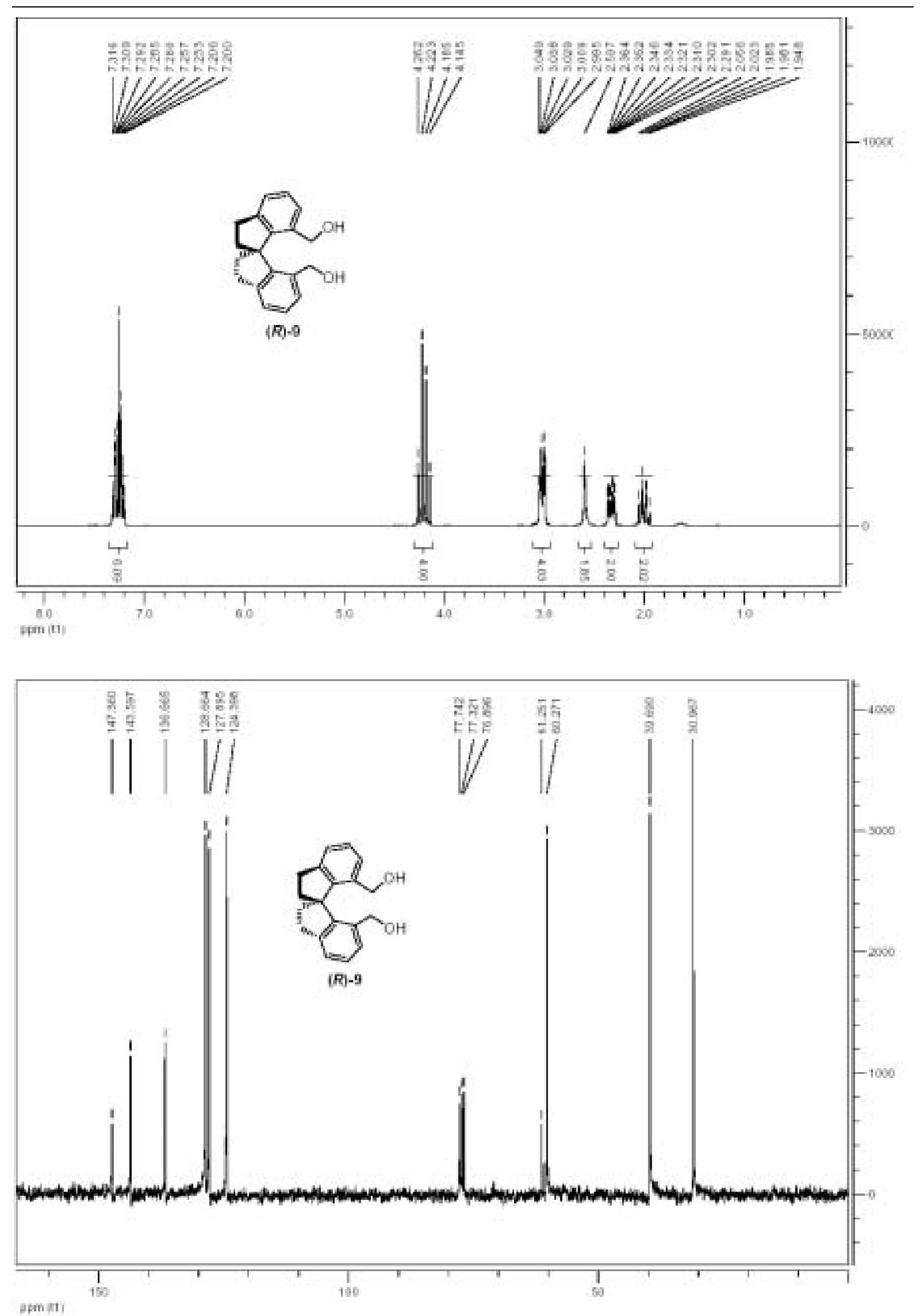

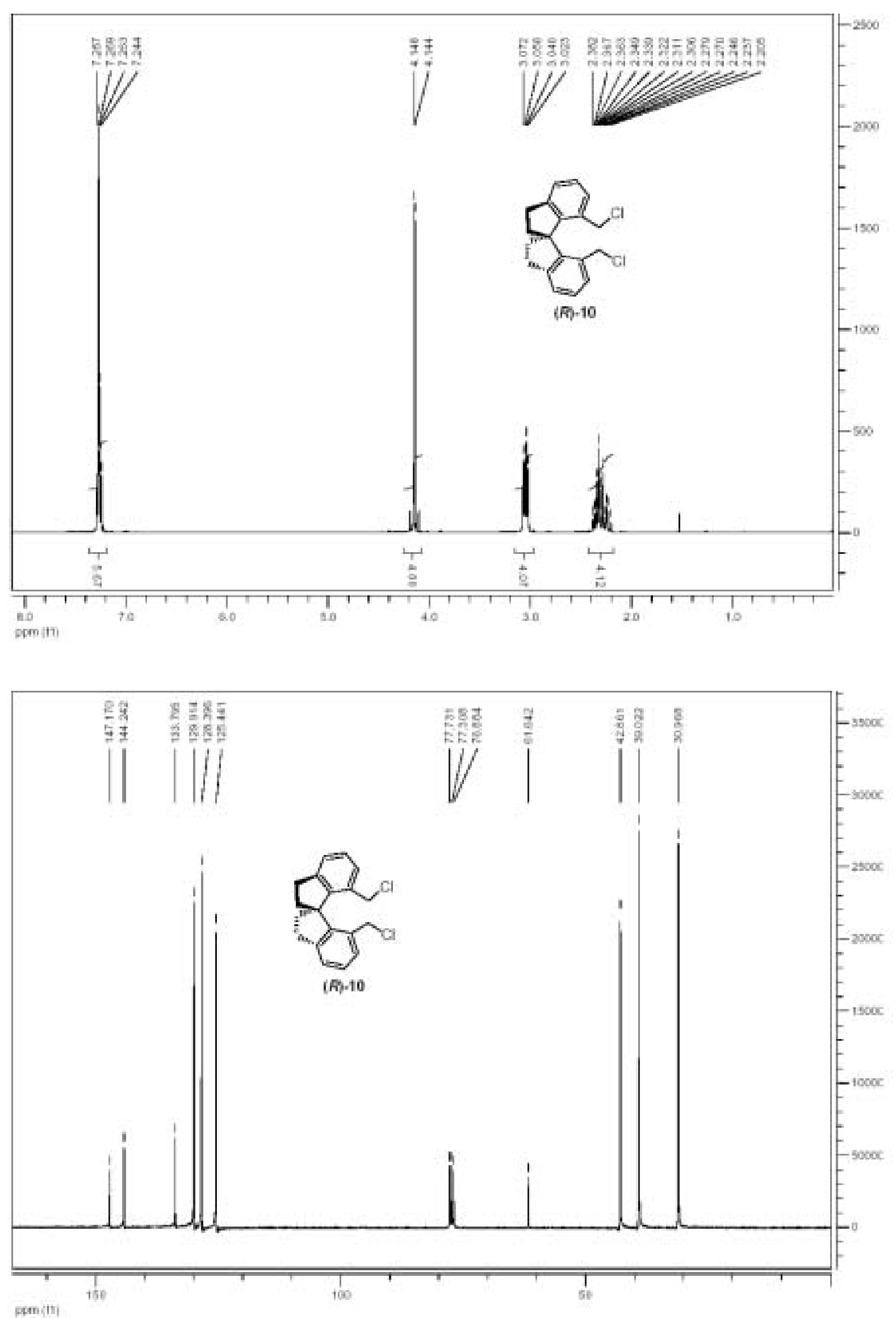

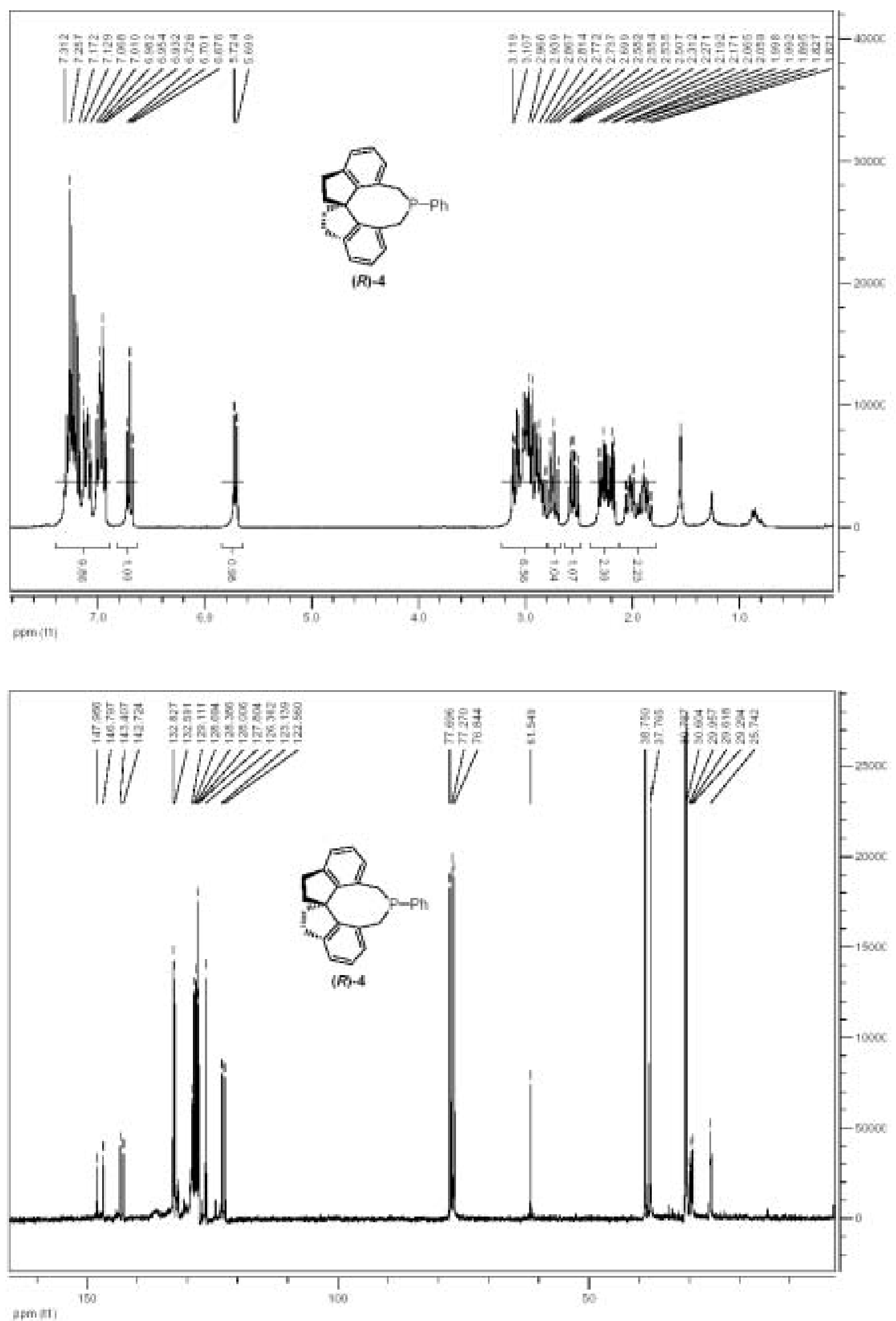

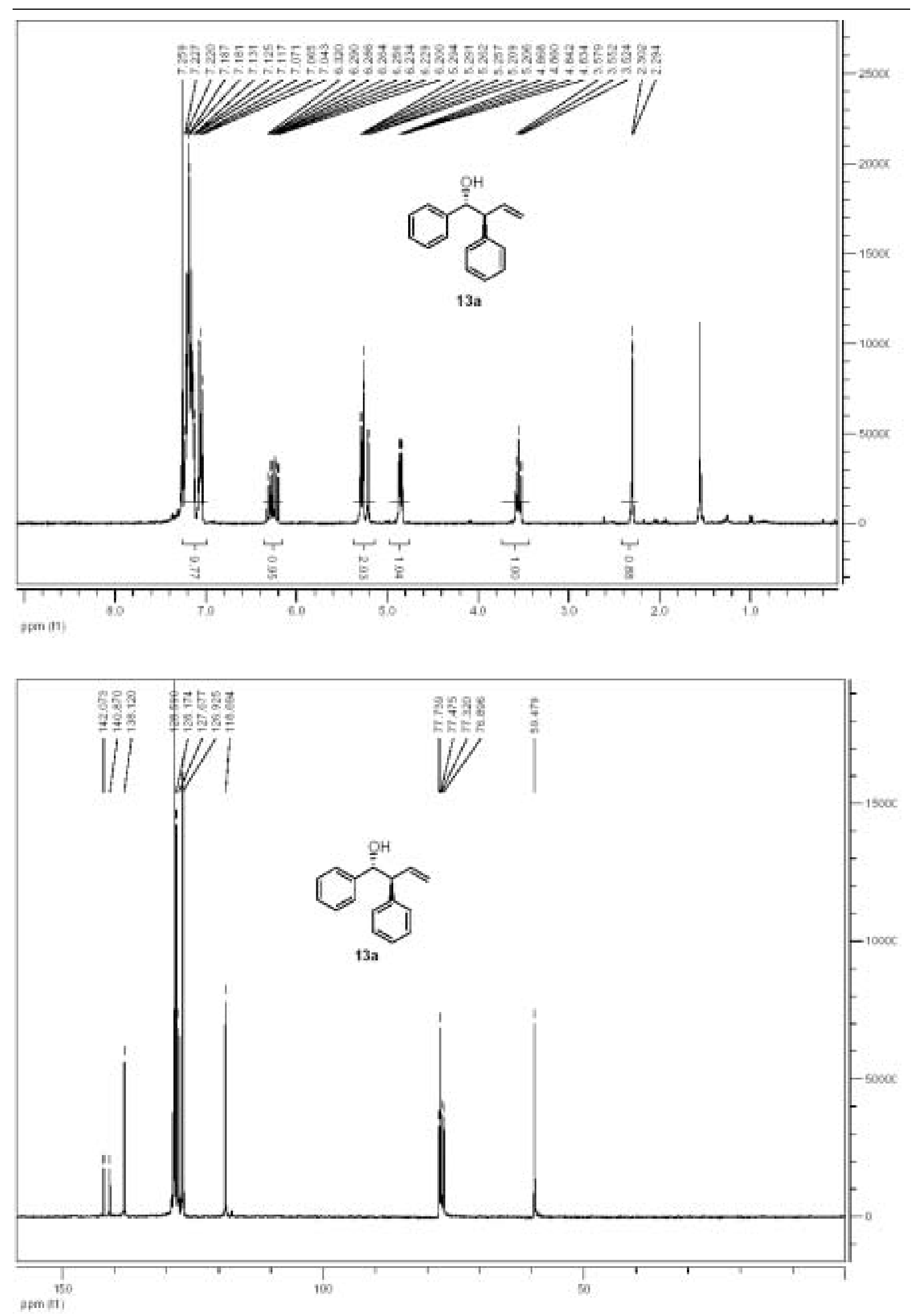

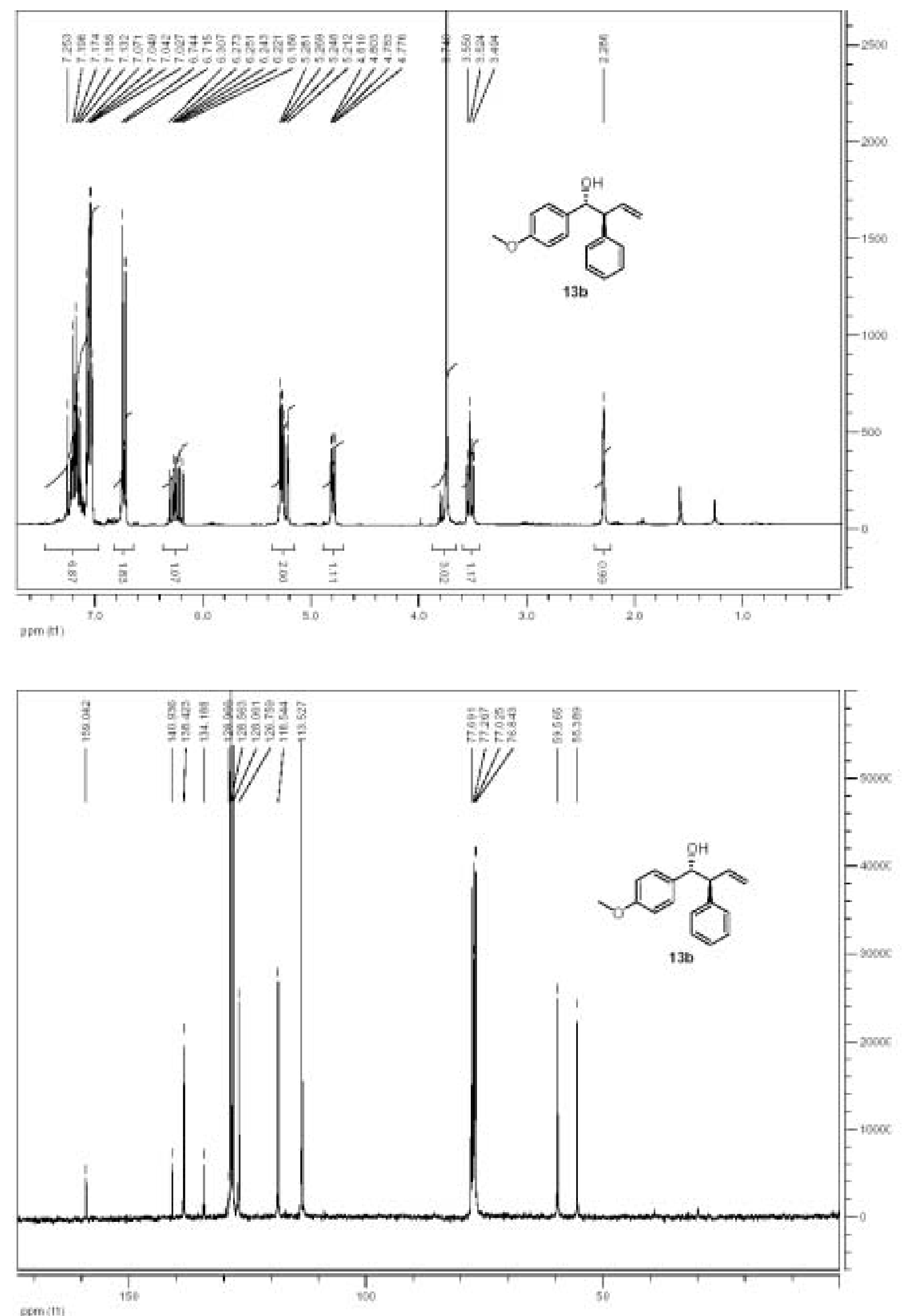

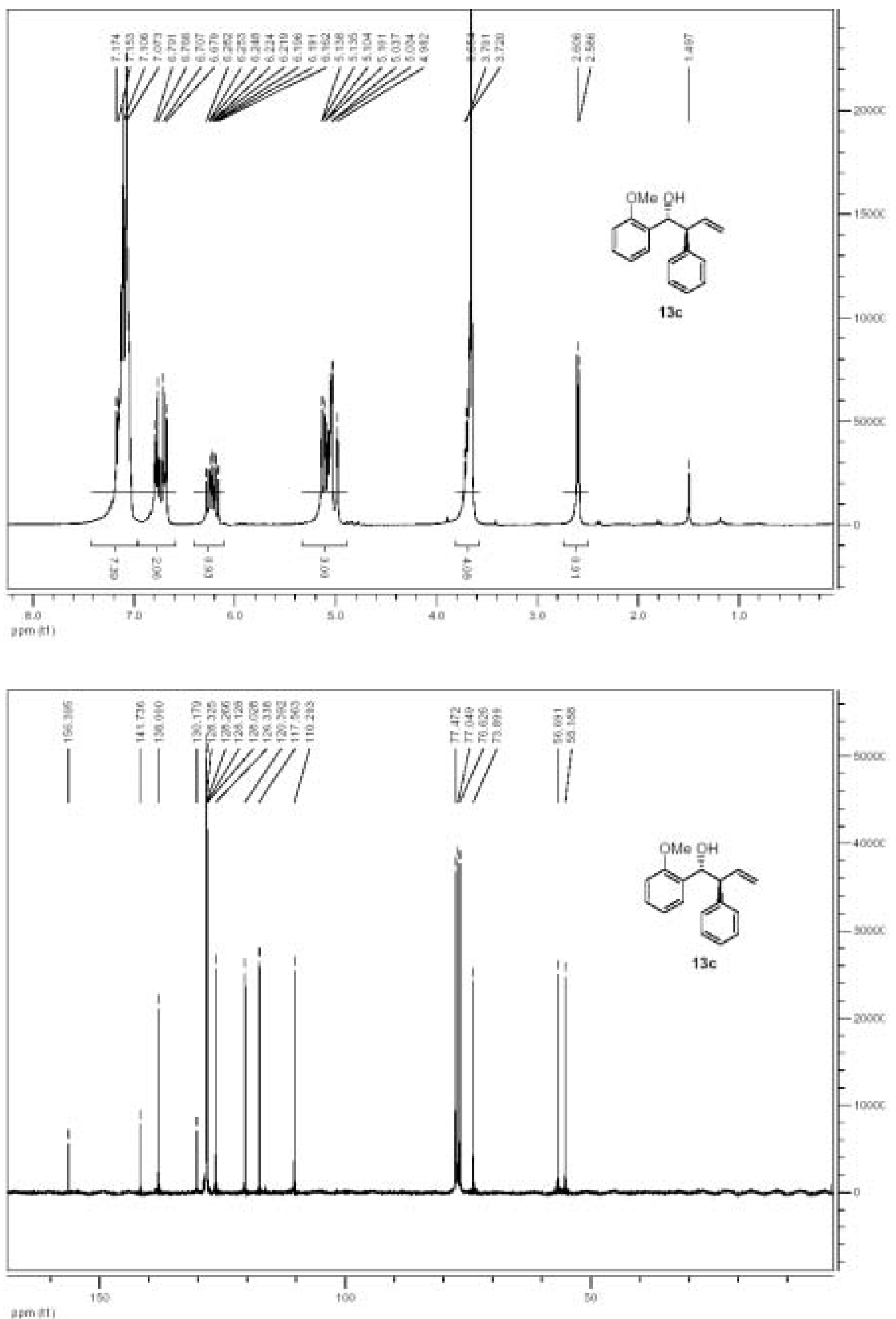

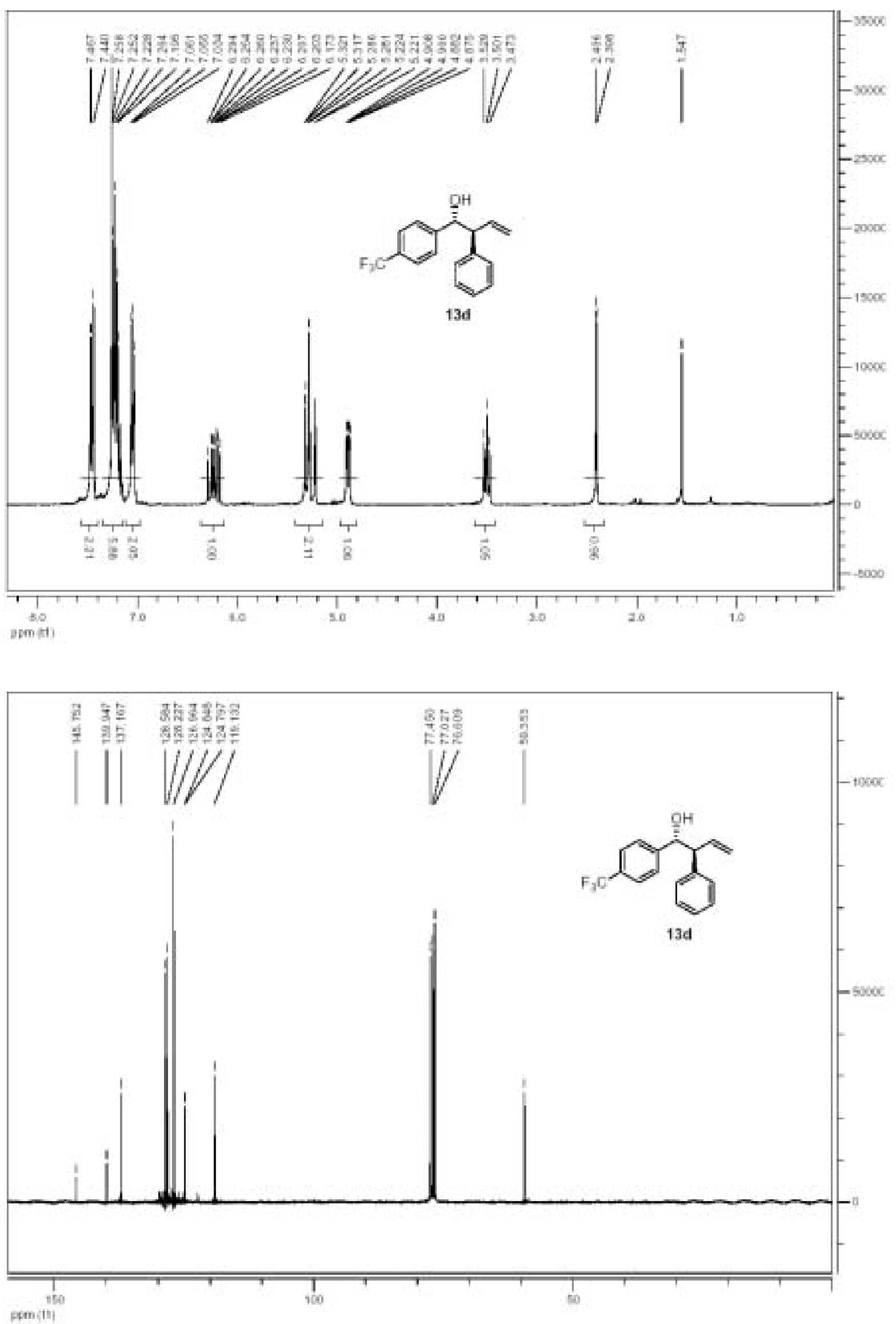

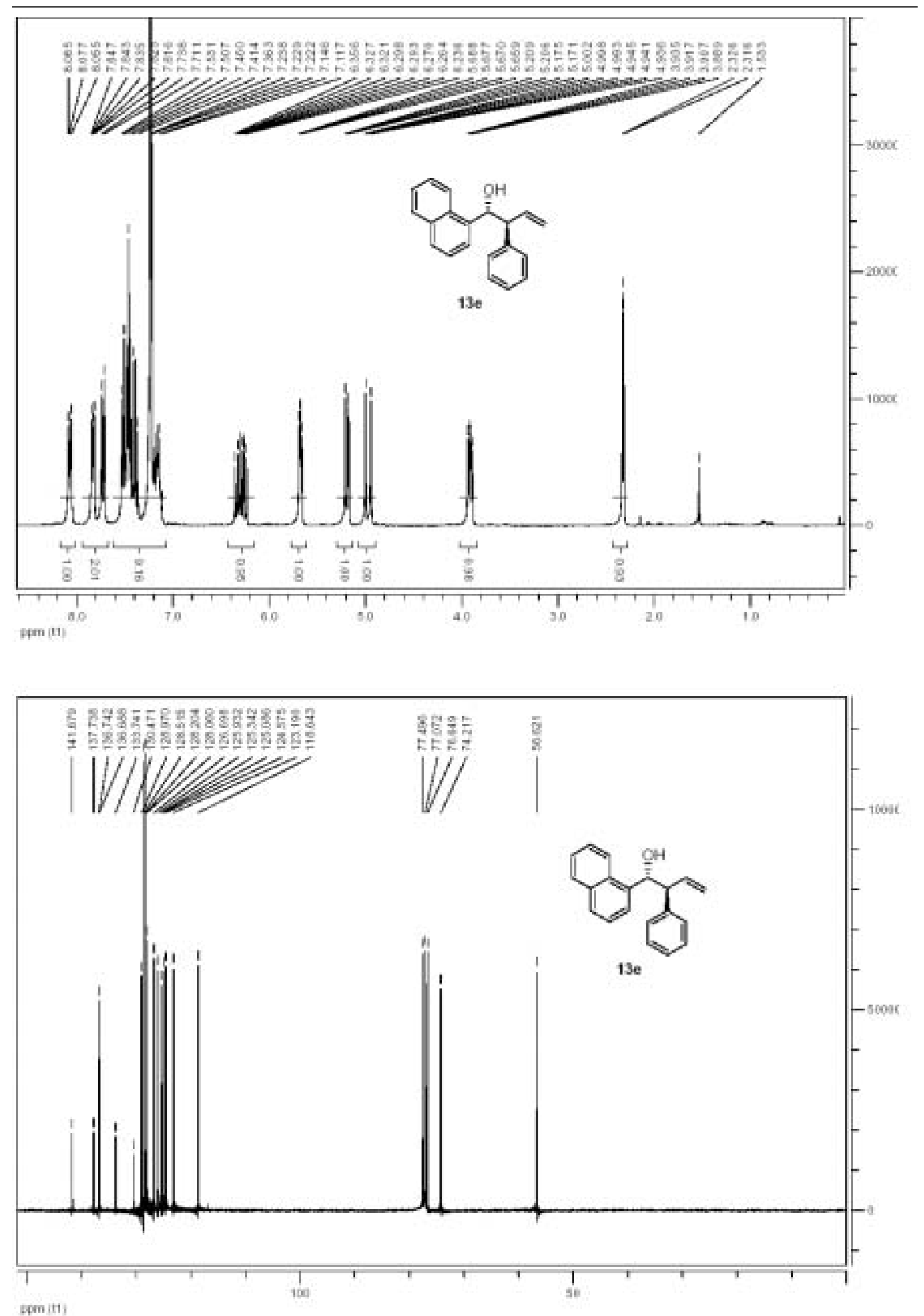

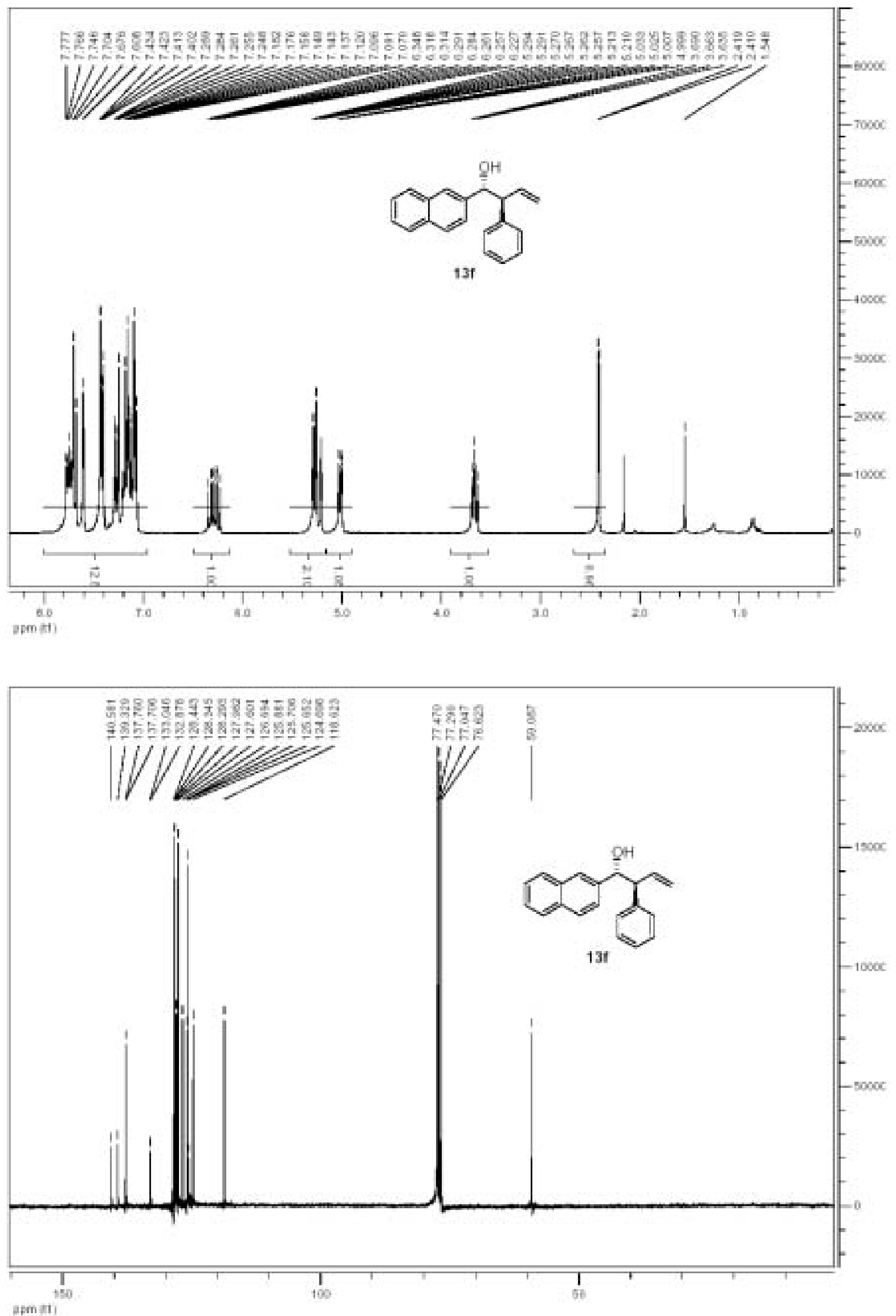

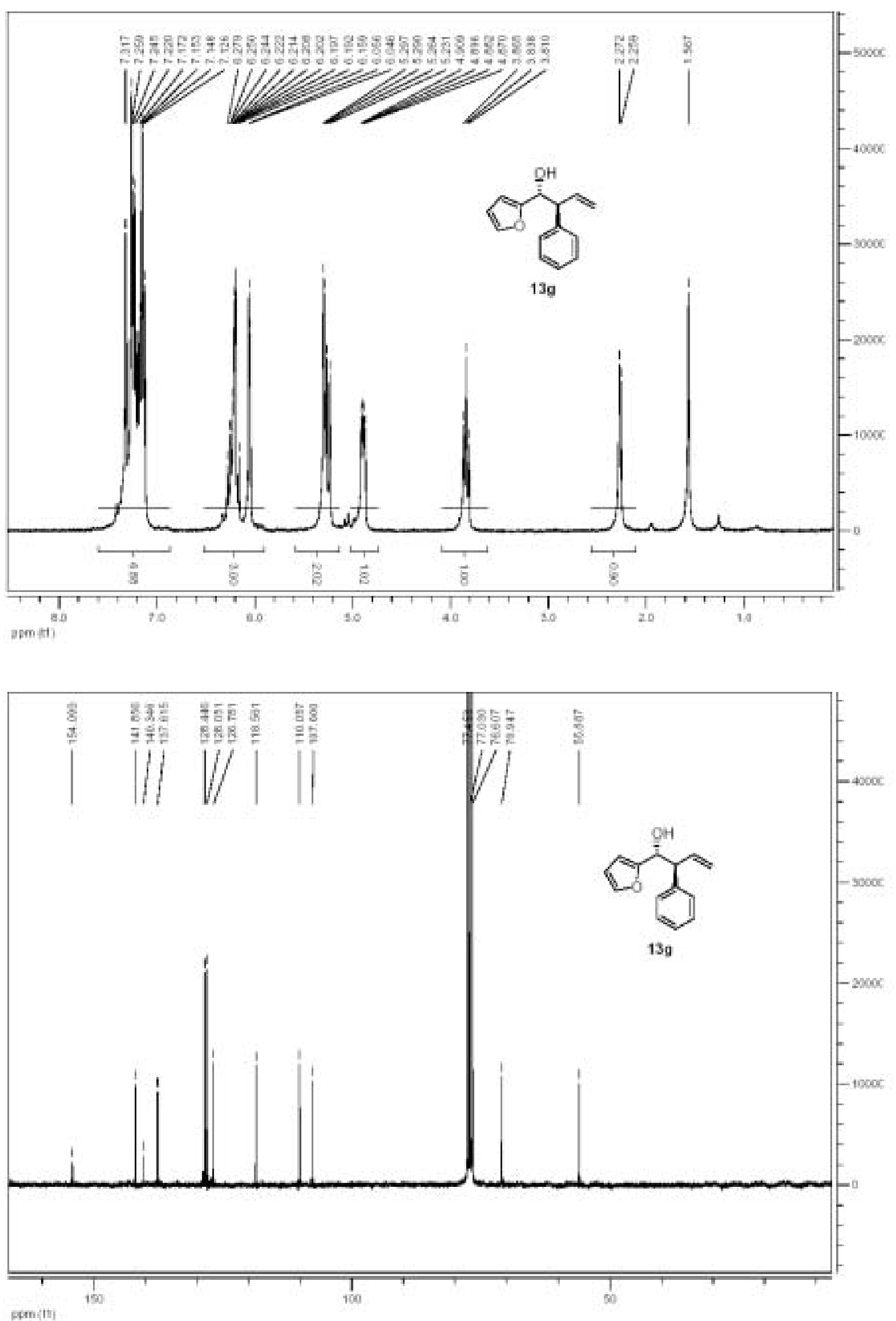

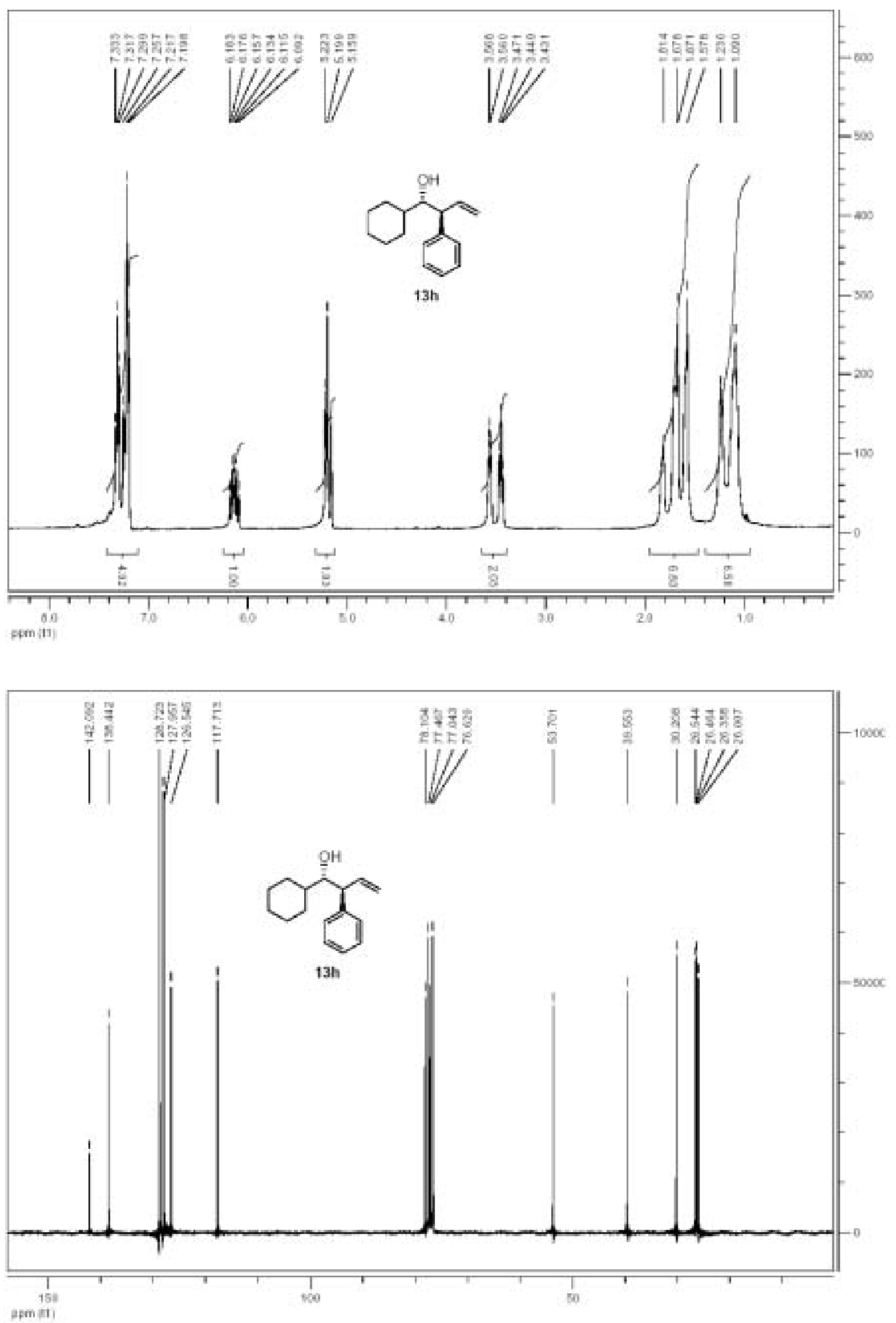

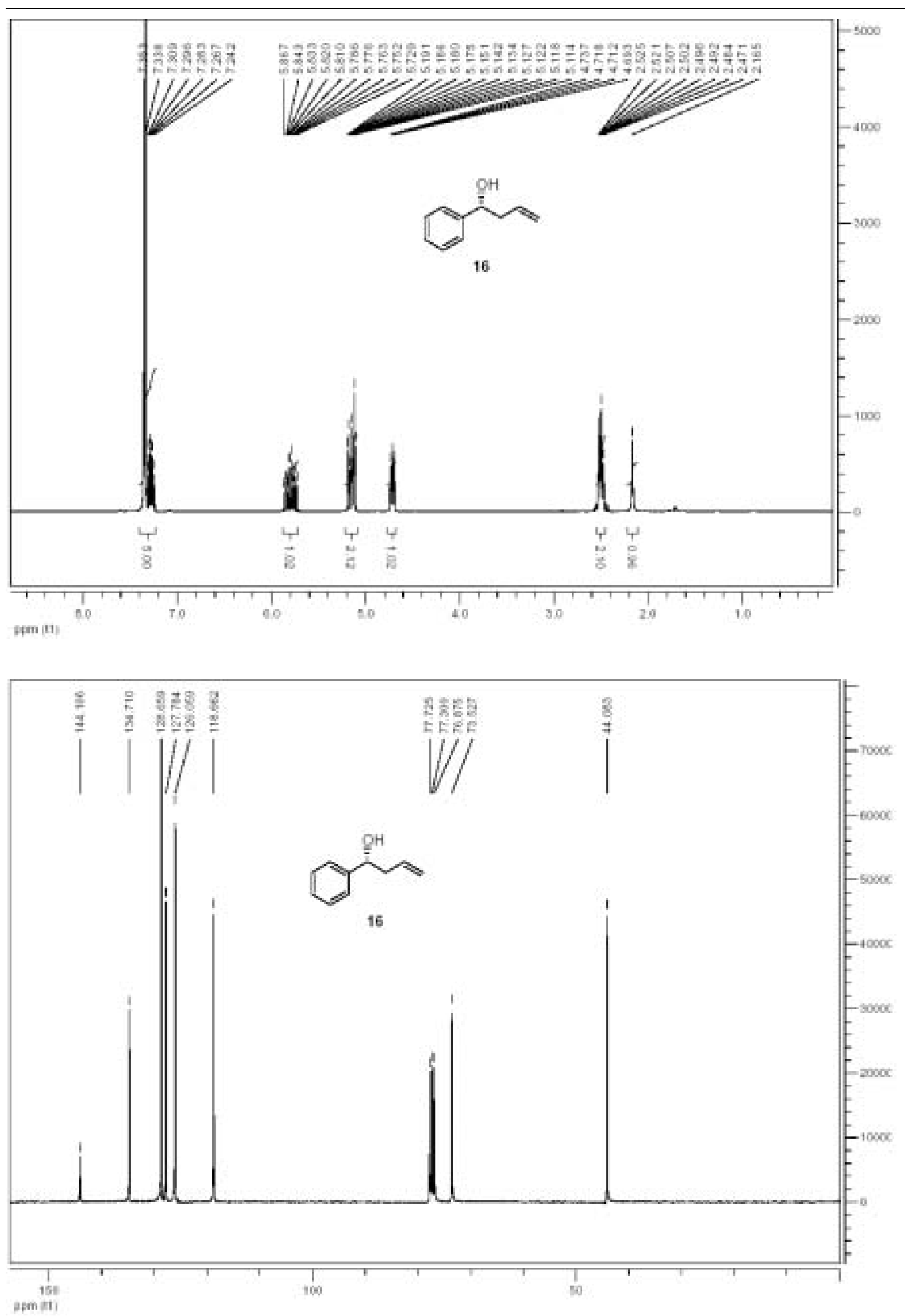